\title{
Isotermas de dessorção e calor isostérico dos frutos de crambe
}

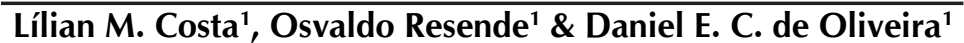

\section{RESUMO}

Neste trabalho objetivou-se determinar as isotermas de sorção dos frutos de crambe e obter os valores do calor isostérico de dessorção em função do teor de água de equilíbrio. Para obtenção do teor de água de equilíbrio higroscópico utilizaram-se os frutos de crambe com teor de água inicial de 26,0 (\% base seca) e o método estático-gravimétrico e, para cada repetição, foram colocados $10 \mathrm{~g}$ de frutos envolvidos por um tecido permeável para permitir o contato do ar com o produto e colocados no interior dos dessecadores enquanto para o controle da umidade relativa no interior dos dessecadores foram utilizadas soluções saturadas. Os dessecadores foram colocados em câmara incubadora tipo B.O.D. regulada para as temperaturas de 25, 30, 35 e $40^{\circ} \mathrm{C}$. O teor de água de equilíbrio higroscópico dos frutos de crambe é diretamente proporcional à atividade de água e decresce com o aumento de temperatura, para um mesmo valor de umidade relativa de equilíbrio. Baseando-se em parâmetros estatísticos os modelos de Copace e Oswin Modificado são os que melhor representam a higroscopicidade dos frutos de crambe quando comparados com os demais modelos testados. O calor isostérico aumenta com a diminuição do teor de água de equilíbrio sendo necessária uma quantidade maior de energia para retirar a água dos frutos de crambe.

Palavras-chave: Crambe abyssinica, atividade de água, modelagem matemática

\section{Desorption isotherms and isosteric heat of the fruits of crambe}

\begin{abstract}
This study aimed to adjust different mathematical models to experimental data of hygroscopicity of crambe fruits, selecting one that best represented the phenomenon, and obtain the values of the isosteric heat of desorption as a function of equilibrium moisture content. To obtain an equilibrium moisture content, crambe fruit was used with an initial moisture content of $26.0 \%$ (dry basis) and static gravimetric method. For each replicate $10 \mathrm{~g}$ of fruit were placed surrounded by a permeable fabric to permit air contact with the product and placed within the desiccators. To control the relative humidity inside the desiccators, saturated solutions were used. The desiccators were placed in incubator B.O.D., adjusted to temperatures of 25, 30, 35 and 40 ${ }^{\circ} \mathrm{C}$. The equilibrium moisture content of crambe fruits is directly proportional to water activity and decreases with increasing temperature for the same value of equilibrium relative humidity. Based on statistical parameters, the models of Copace and Modified Oswin are those that best represent the hygroscopicity of crambe fruits compared to other tested models. The isosteric heat increases with decrease in equilibrium moisture content and requires a larger amount of energy to remove water from the crambe fruit.
\end{abstract}

Key words: Crambe abyssinica, water activity, mathematical modeling 


\section{INTRODUÇÃO}

O Crambe abyssinica é uma cultura considerada potencial para a produção industrial de biodiesel em função das características de cultivo e alta proporção de óleo. Estudo realizado com crambe cultivado no Mato Grosso do Sul com a variedade FMS brilhante, indicou 40\% de lipídeos (Souza et al., 2009). O interesse pelo crambe surgiu em virtude da sua superioridade em relação à soja na produção de óleos vegetais e por se adaptar com facilidade ao plantio direto. Frisa-se que um dos maiores problemas que a produção de biodiesel vem enfrentando é a falta de matéria-prima e o crambe, por ser uma cultura totalmente mecanizada e de baixo custo, poderá ser produzido em larga escala e rapidamente sem grandes investimentos por parte dos produtores rurais. $\mathrm{O}$ custo do óleo vegetal corresponde a cerca de $85 \%$ do custo do biodiesel (Guirra, 2009) quando este é produzido em plantas de alta capacidade.

Pode-se ressaltar, então, o interesse em se obter uma matériaprima para a produção de biodiesel de custo menos elevado e que tenha bom desempenho agronômico. Contudo, o cultivo desta oleaginosa em solo brasileiro somente terá sucesso se os patamares de produtividade forem satisfatórios e, neste caso, o uso de sementes de qualidade superior certamente contribuirá decisivamente para que as metas de produção sejam alcançadas (Neves et al., 2007). Neste contexto o procedimento de secagem das sementes faz-se necessário para garantir posteriormente, no campo, a qualidade da cultura durante o armazenamento e o seu bom desempenho.

As condições de umidade relativa e de temperatura durante o armazenamento, em que os produtos alcançarão o equilíbrio higroscópico específico, determinarão a manutenção de sua qualidade fisiológica, por maior ou menor tempo (Borges et al., 2009).

A relação entre o teor de água de equilíbrio e a umidade relativa de equilíbrio também designada atividade da água, constitui fator essencial nos projetos e estudos de sistemas de secagem, manuseio, armazenagem, embalagem e transporte e na modelagem da longevidade das sementes (Araújo et al., 2001).

$\mathrm{O}$ conceito de atividade de água indica a intensidade das forças que unem a água com outros componentes não aquosos e, consequentemente, a água disponível para o crescimento de micro-organismos podendo ocorrer diferentes reações químicas e bioquímicas (Ordoñez, 2005). Os produtos agrícolas possuem a propriedade de realizar trocas de água na forma de vapor, com o ambiente que os envolve. Essas trocas podem ocorrer por meio do ganho ou da perda de água, fenômenos conhecidos, respectivamente, por adsorção e dessorção, de acordo com as características higroscópicas recíprocas do produto e do ar. A afinidade entre a água e os outros componentes (gordura, amido, açúcar, proteínas, etc.) de um produto define sua higroscopicidade (Brooker et al., 1992).

Vários pesquisadores têm estudado o comportamento higroscópico de diversos produtos agrícolas que descrevem modelos matemáticos distintos para expressar o teor de água de equilíbrio em função da temperatura e umidade relativa do ar, como: para o nabo forrageiro (Sousa et al., 2013), quiabo (Goneli et al., 2010), arroz vermelho (Bastos, 2009), mamona (Goneli et al., 2008), feijão vermelho (Resende et al., 2006b), milho em espiga (Corrêa et al., 2005b), dentre outros. Entretanto, para o estabelecimento de isotermas que representam esta relação de equilíbrio são utilizados modelos matemáticos empíricos, uma vez que nenhum modelo teórico desenvolvido tem sido capaz de estimar com precisão o teor de água de equilíbrio para uma ampla faixa de temperatura e umidade relativa do ar (Corrêa et al., 2005b).

As isotermas de equilíbrio podem ser determinadas por meio de métodos gravimétrico e higrométrico. No método higrométrico a umidade do material é mantida constante devido à variação da umidade relativa do ar circundante, até que esta umidade relativa atinja um valor constante de equilíbrio, sendo esta medida via higrômetro. Já no método gravimétrico a temperatura do ar e a atividade de água são mantidas constantes até que o teor de água da amostra atinja o valor de equilíbrio. $\mathrm{O}$ ar pode ser circulado (método dinâmico) ou estar estagnado (método estático). O método estático possui as vantagens de se obter condições termodinâmicas constantes com maior facilidade e permitir o uso de soluções ácidas ou soluções salinas saturadas (Mortola et al., 2003).

Segundo Lima et al. (2008) o calor isostérico é essencial nos estudos de secagem e armazenamento de produtos agrícolas servindo para estimar as necessidades energéticas do processo de secagem fornecendo, também, dados sobre o estado da água no produto; é um bom parâmetro para se estimar a quantidade mínima de calor requerida para remover uma quantidade de água e permite algumas deduções sobre a microestrutura e as mudanças físicas que ocorrem na superfície dos alimentos.

O calor isostérico é um importante parâmetro a ser obtido haja vista que por meio dele é possível se ter uma idéia sobre a demanda energética nos processos de desidratação e secagem de materiais biológicos. De acordo com Aviara \& Ajibola (2002) o calor isostérico é uma propriedade termodinâmica de sorção de água crucial na análise do fenômeno de transferência de calor e de massa.

$\mathrm{O}$ conhecimento do calor isostérico em função do teor de água de equilíbrio é essencial nos estudos de secagem e armazenamento de produtos agrícolas servindo para estimar as necessidades energéticas do processo de secagem além de fornecer dados sobre o estado da água no produto. Ante o exposto o objetivo neste trabalho foi determinar as isotermas de sorção dos frutos de crambe e obter os valores do calor isostérico de dessorção em função do teor de água de equilíbrio.

\section{Materal e Métodos}

O presente trabalho foi desenvolvido no Laboratório de Pós-colheita de Produtos Vegetais do Instituto Federal de Educação, Ciência e Tecnologia Goiano - Câmpus Rio Verde (IF Goiano - Câmpus Rio Verde) localizado no município de Rio Verde, GO.

Visando à condução do experimento, foram utilizados frutos de crambe cultivados na safra de inverno de 2009, colhidas manualmente na área experimental do IF Goiano - Câmpus Rio Verde, com teor de água de 26,0 (\% b.s.).

Os teores de água dos frutos foram determinados por gravimetria utilizando-se a estufa a $105 \pm 3{ }^{\circ} \mathrm{C}$, durante $24 \mathrm{~h}$, em duas repetições (Brasil, 2009). 
Para obtenção do teor de água de equilíbrio higroscópico utilizaram-se frutos com teor de água inicial de 26,0 (\% b.s.) e o método estático-gravimétrico; para cada repetição foram colocados $10 \mathrm{~g}$ de frutos envolvidos por um tecido permeável (voile) a fim de permitir a passagem do ar através do produto e só então colocados no interior dos dessecadores. A temperatura e a umidade relativa do ar foram monitoradas por meio de um data logger colocado no interior dos dessecadores; Visando ao controle da umidade relativa no interior dos dessecadores utilizaram-se soluções saturadas de diferentes sais, apresentados na Tabela 1, preparadas de acordo com a metodologia descrita por Dhingra \& Sinclair (1995); os dessecadores foram colocados em câmara incubadora tipo B.O.D. (Biochemical Oxigen Demand) reguladas para as temperaturas de $25,30,35$ e $40^{\circ} \mathrm{C}$.

Tabela 1. Umidades relativas (decimal) estabelecidas no interior dos dessecadores para determinação do equilíbrio higroscópico dos frutos de crambe, pelo método estático-gravimétrico

\begin{tabular}{|c|c|c|c|c|c|}
\hline \multirow{2}{*}{\multicolumn{2}{|c|}{ Composto químico }} & \multicolumn{4}{|c|}{ Temperatura $\left({ }^{\circ} \mathrm{C}\right)$} \\
\hline & & 25 & 30 & 35 & 40 \\
\hline $\mathrm{LiCl}$ & Cloreto de lítio & 0,11 & 0,15 & 0,14 & 0,11 \\
\hline $\mathrm{CaCl}_{2}$ & Cloreto de cálcio & 0,30 & 0,27 & 0,21 & 0,20 \\
\hline $\mathrm{Ca}\left(\mathrm{NO}_{3}\right)_{2}$ & Nitrato de cálcio & 0,46 & 0,46 & 0,44 & 0,45 \\
\hline $\mathrm{NaCl}$ & Cloreto de sódio & 0,74 & 0,74 & 0,74 & 0,74 \\
\hline $\mathrm{KBr}$ & Brometo de potássio & 0,81 & - & - & - \\
\hline
\end{tabular}

Durante o processo as amostras foram pesadas periodicamente e o equilíbrio higroscópio foi atingido quando a variação da massa permaneceu invariável durante aproximadamente três pesagens consecutivas, após o que foram determinados os teores de água em estufa a $105 \pm 3^{\circ} \mathrm{C}$, durante $24 \mathrm{~h}$ (Brasil, 2009).

Aos dados experimentais do teor de água de equilíbrio foram ajustados os modelos matemáticos frequentemente utilizados para representação da higroscopicidade de produtos agrícolas, cujas expressões estão apresentadas na Tabela 2.

Tabela 2. Modelos matemáticos utilizados para predizer o fenômeno de higroscopicidade de produtos agrícolas

\begin{tabular}{|c|c|c|}
\hline Designação do modelo & Modelo & \\
\hline$X e^{*}=\left\{1 /\left[\left(1-a_{w}\right) \cdot(1 / a \cdot b+((a-1) / a \cdot b))\right]\right\}$ & BET & $(1)$ \\
\hline$X e^{*}=a-b \cdot \ln \left[-(T+c) \cdot \ln \left(a_{w}\right)\right]$ & ChungPfost & $(2)$ \\
\hline$X e^{*}=\exp \left[a-(b \cdot T)+\left(c \cdot a_{w}\right)\right]$ & Copace & (3) \\
\hline$X e^{*}=\left(a \cdot b \cdot c \cdot a_{w}\right) /\left[\left(1-c \cdot a_{w}\right) \cdot\left(1-c \cdot a_{w}+b \cdot c \cdot a_{w}\right)\right]$ & ]GAB & $(4)$ \\
\hline$X e^{*}=\left(\exp (a-b \cdot T) /-\ln \left(a_{w}\right)\right)^{1 / c}$ & $\begin{array}{l}\text { Halsey } \\
\text { Modificado }\end{array}$ & (5) \\
\hline$X e^{*}=\left\{\ln \left(1-a_{w}\right) /[-a \cdot(T+b)]\right\}^{1 / c}$ & $\begin{array}{l}\text { Henderson } \\
\text { Modificado }\end{array}$ & (6) \\
\hline$X e^{*}=\left[\ln \left(1-a_{w}\right) /(-a \cdot T+273,16)\right]^{1 / c}$ & Henderson & (7) \\
\hline$X e^{*}=(a+b T) /\left[a_{w} /\left(1-a_{w}\right)\right]^{1 / c}$ & $\begin{array}{l}\text { Oswin } \\
\text { Modificado }\end{array}$ & (8) \\
\hline $\begin{array}{l}X e^{*}=a \cdot\left(a_{w} \cdot b / T^{c}\right) \\
X e^{*}=\exp \left\{a-(b \cdot T)+\left[c \cdot \exp \left(a_{w}\right)\right]\right\}\end{array}$ & $\begin{array}{l}\text { Sabbah } \\
\text { Sigma Copace }\end{array}$ & $\begin{array}{l}(9) \\
(10) \\
\end{array}$ \\
\hline
\end{tabular}

em que:

$\mathrm{Xe}^{*}$ - teor de água de equilíbrio, \% b.s

$\mathrm{a}_{\mathrm{w}} \quad$ - atividade de água, decimal

$\mathrm{T}$ - temperatura, ${ }^{\circ} \mathrm{C}$

$\mathrm{a}, \mathrm{b}, \mathrm{c}$ - coeficientes que dependem do produto
Realizou-se, visando ao ajuste dos modelos matemáticos, a análise de regressão não linear, pelo método Gauss Newton, utilizando-se um programa estatístico; para verificar o grau de ajuste de cada modelo foi considerada a significância do coeficiente de regressão pelo teste t adotando-se o nível de 5\% de significância, a magnitude do coeficiente de determinação $\left(\mathrm{R}^{2}\right)$, do erro médio estimado (SE), os valores do erro médio relativo $(\mathrm{P})$ e o teste de Qui-quadrado $\left(\chi^{2}\right)$ a nível de significância de $1 \%$ e o intervalo de confiança a $99 \%$ (P< $0,01)$. Considerou-se o valor do erro médio relativo inferior a 10\% como um dos critérios para a seleção dos modelos, de acordo com Mohapatra \& Rao (2005). O erro médio estimado e relativo bem como o teste de Qui-quadrado para cada um dos modelos, foram calculados conforme as seguintes expressões, respectivamente:

$$
\begin{aligned}
& \mathrm{SE}=\sqrt{\frac{\sum(\mathrm{Y}-\hat{\mathrm{Y}})^{2}}{\mathrm{GLR}}} \\
& \mathrm{P}=\frac{100}{\mathrm{n}} \sum \frac{|\mathrm{Y}-\hat{\mathrm{Y}}|}{\mathrm{Y}} \\
& \chi^{2}=\sum \frac{(\mathrm{Y}-\hat{\mathrm{Y}})^{2}}{\mathrm{GLR}}
\end{aligned}
$$

em que:

Y - valor observado experimentalmente

$\hat{Y} \quad$ - valor calculado pelo modelo

$\mathrm{n} \quad$ - número de observações experimentais

GLR - graus de liberdade do modelo (número de observações menos o número de parâmetros do modelo)

Os valores do calor isostérico líquido de sorção (ou entalpia diferencial) para cada teor de água de equilíbrio, foram obtidos por meio da equação Clausius-Clayperon (Iglesias \& Chirife, 1976) como indicado a seguir:

$$
\frac{\partial \operatorname{In}\left(\mathrm{a}_{\mathrm{w}}\right)}{\partial \mathrm{T}}=\frac{\Delta \mathrm{h}_{\mathrm{st}}}{\mathrm{RT}_{\mathrm{a}}^{2}}
$$

em que:

$\mathrm{T}_{\mathrm{a}}$ - temperatura absoluta, $\mathrm{K}$

$\Delta \mathrm{h}_{\mathrm{st}}$ - calor isostérico líquido de sorção, $\mathrm{kJ} \mathrm{kg}^{-1}$

$\mathrm{R}$ - constante universal dos gases, $8,314 \mathrm{~kJ} \mathrm{kmol}^{-1} \mathrm{~K}^{-1}$

sendo para o vapor d'água $0,4619 \mathrm{~kJ} \mathrm{~kg}^{-1} \mathrm{~K}^{-1}$

Integrando a Eq. 14 e assumindo que o calor isostérico líquido de sorção é independente da temperatura, obtém-se o calor isostérico líquido de sorção para cada teor de água de equilíbrio, conforme a Eq. 15 (Wang \& Brennan, 1991):

$$
\operatorname{In}\left(\mathrm{a}_{\mathrm{w}}\right)=-\left(\frac{\Delta \mathrm{h}_{\mathrm{st}}}{\mathrm{R}}\right) \cdot \frac{1}{\mathrm{~T}_{\mathrm{a}}}+\mathrm{C}
$$

em que:

C - coeficiente do modelo 
Os valores de atividade de água, temperatura e teor de água de equilíbrio foram obtidos a partir das isotermas de dessorção dos frutos de crambe utilizando-se o modelo de melhor ajuste aos dados experimentais. Obteve-se o calor isostérico integral de sorção adicionando-se aos valores de calor isostérico líquido de sorção, o valor do calor latente de vaporização da água livre, de acordo com a Eq. 16:

$$
\mathrm{Q}_{\mathrm{st}}=\Delta \mathrm{h}_{\mathrm{st}}+\mathrm{L}=\mathrm{a} \cdot \exp \left(-\mathrm{b} \cdot \mathrm{Xe}^{*}\right)+\mathrm{L}
$$

em que:

$\mathrm{Q}_{\mathrm{st}} \quad$ - calor isostérico integral de sorção, $\mathrm{kJ} \mathrm{kg}^{-1}$

$\mathrm{L}$ - calor latente de vaporização da água livre, $\mathrm{kJ} \mathrm{kg}^{-1}$

$\mathrm{a}, \mathrm{b}$ - coeficientes do modelo

O calor latente de vaporização da água livre (L) em kJ kg-1, necessário ao cálculo de $\mathrm{Q}_{\mathrm{st}}$, foi obtido através da temperatura média (T) na faixa em estudo, em ${ }^{\circ} \mathrm{C}$, por meio da seguinte equação:

$$
\mathrm{L}=2502,2-2,39 \mathrm{~T}
$$

\section{Resultados E Discussão}

Os valores médios do teor de água de equilíbrio higroscópico dos frutos de crambe obtidos por dessorção para as temperaturas de $25,30,35$ e $40{ }^{\circ} \mathrm{C}$ e atividade de água entre 0,11 a 0,81 (decimal) são apresentados na Tabela 3. Verifica-se, para uma mesma temperatura, que com a variação da atividade de água tem-se um aumento sensível do teor de água de equilíbrio. Nota-se que com o aumento da temperatura o teor de água de equilíbrio decresce para os frutos de crambe; condição semelhante também foi constatada por Corrêa et al. (2005c) ao estudarem o teor de água de equilíbrio higroscópico das espigas de milho da cultivar BR 205 que decresce com o aumento de temperatura para uma mesma umidade relativa, seguindo a tendência da maioria dos produtos agrícolas já estudados.

Na Tabela 4 estão apresentados os parâmetros dos modelos ajustados aos valores de teor de água de equilíbrio higroscópico para os frutos de crambe obtidos por dessorção, pelo método estático para diferentes condições de temperatura e atividades de água.

Verifica-se, na Tabela 4, que os modelos matemáticos utilizados para descrever a higroscopicidade dos frutos de
Tabela 3. Valores médios do teor de água de equilíbrio (\% b.s.) dos frutos de crambe obtidos pelo processo de dessorção, em função da temperatura $\left({ }^{\circ} \mathrm{C}\right)$ e atividade de água (decimal)

\begin{tabular}{ccccc}
\hline Atividade de & \multicolumn{4}{c}{ Temperatura $\left({ }^{\circ} \mathbf{C}\right)$} \\
\cline { 2 - 5 } água (decimal) & $\mathbf{2 5}$ & $\mathbf{3 0}$ & $\mathbf{3 5}$ & $\mathbf{4 0}$ \\
0,11 & 1,2 & - & - & 0,8 \\
0,14 & - & - & 1,0 & - \\
0,15 & - & 1,1 & - & - \\
0,20 & - & - & - & 1,2 \\
0,21 & - & - & 1,4 & - \\
0,27 & - & 1,4 & - & - \\
0,30 & 1,8 & - & - & - \\
0,44 & - & - & 2,0 & \\
0,45 & - & - & - & 1,4 \\
0,46 & 2,2 & 2,0 & - & - \\
0,74 & 3,7 & 3,3 & 3,0 & 1,7 \\
0,81 & 4,3 & - & - & - \\
\hline
\end{tabular}

crambe apresentaram, para a maioria dos seus coeficientes, significância de regressão a nível de 0,01 de probabilidade pelo teste $t$; de modo geral, os modelos apresentaram valores elevados do coeficiente de determinação, superiores a 0,90 , exceto os modelos de BET, GAB e Henderson Modificado, que ficaram abaixo de $80 \%$. Para uma análise mais detalhada foram utilizados outros parâmetros estatísticos para respaldar a seleção do melhor modelo; segundo Madamba et al. (1996) a utilização do coeficiente de determinação como o único critério de avaliação para a seleção dos modelos não lineares, não constitui um bom parâmetro para representação do fenômeno.

De acordo com os valores obtidos referentes ao erro médio estimado (SE) nota-se que os modelos Copace, Oswin Modificado e Sigma Copace, apresentaram menores valores quando comparados com os demais.

Nota-se, inda na Tabela 4, que os valores do erro médio relativo $(\mathrm{P})$ foram inferiores a $10 \%$ somente para os modelos de Copace, Halsey Modificado, Oswin Modificado e Sigma Copace que, de acordo com Mohapatra \& Rao (2005) indica uma representação adequada do fenômeno estudado.

Em relação ao teste de Qui-quadrado $\left(\chi^{2}\right)$ os dez modelos analisados se encontram no intervalo de confiança de $95 \%$; entretanto, comparando a magnitude dos valores somente os modelos de Copace, Halsey Modificado, Henderson Modificado, Oswin modificado, Sabbah e Sigma Copace apresentaram menores valores em comparação aos demais.

Tabela 4. Coeficientes dos modelos ajustados aos teores de água de equilíbrio higroscópico para os frutos de crambe com seus respectivos coeficientes de determinação $\left(R^{2}\right)$, erros médios estimado (SE) e erros médios relativo $(P)$ e

\begin{tabular}{|c|c|c|c|c|c|c|c|}
\hline \multirow{2}{*}{ Modelos } & \multicolumn{3}{|c|}{ Coeficientes } & \multirow{2}{*}{$\mathbf{R}^{2}$} & \multirow{2}{*}{$\begin{array}{c}\text { SE } \\
\text { Decimal }\end{array}$} & \multirow{2}{*}{$\begin{array}{c}P \\
(\%)\end{array}$} & \multirow{2}{*}{$\begin{array}{c}\chi^{2} \\
\text { Decimal } \times 10^{-5}\end{array}$} \\
\hline & $a$ & b & C & & & & \\
\hline Chung Pfost & $0,0476^{* *}$ & $0,0103 * *$ & $13,94110^{\star \star}$ & 0,8917 & 0,004 & 15,78 & 1,30 \\
\hline GAB & $0,01217^{* *}$ & $-39,38827^{\mathrm{NS}}$ & $0,840090^{\star *}$ & 0,7860 & 0,005 & 16,19 & 2,56 \\
\hline Halsey Modificado & $-6,41978^{* *}$ & $0,06636^{* *}$ & $2,086850 * *$ & 0,9476 & 0,003 & 9,14 & 0,62 \\
\hline Henderson Modificado & $118,8274^{\text {NS }}$ & $-15,0907^{\star *}$ & $2,067500 * *$ & 0,7849 & 0,005 & 14,80 & 0,24 \\
\hline Sabbah & $1,426837^{\mathrm{NS}}$ & $0,673181^{* \star}$ & $1,063731^{\star *}$ & 0,9188 & 0,003 & 14,48 & 0,97 \\
\hline Sigma Copace & $-4,42807^{\star \star}$ & $0,03274^{* *}$ & 0,938990 ** & 0,9459 & 0,002 & 9,50 & 0,62 \\
\hline
\end{tabular}
Qui - quadrado $\left(\chi^{2}\right)$

${ }^{* *}$ Significativo a 0,01 pelo teste t, " Significativo a 0,05 pelo teste t, NS Não significativo 
Recomendam-se, desta forma, dois modelos para a descrição do teor de água de equilíbrio higroscópico para os frutos de crambe Copace e Oswin Modificado; já Espíndola (2007) obteve melhor ajuste do modelo de Peleg em estudos de processo de adsorção de sementes da espécie arbórea Cedrela fissilis e de sementes de feijoeiro cultivar Una (Francisco et al., 2007) na temperatura de $25^{\circ} \mathrm{C}$.

Na Figura 1 estão apresentados os valores experimentais do teor de água de equilíbrio dos frutos de crambe, obtidos por dessorção, bem como suas isotermas estimadas pelo modelo de Copace. Verifica-se, ainda, que para uma atividade de água constante os valores de teor de água de equilíbrio higroscópico dos frutos de crambe diminuíram com o aumento da temperatura.

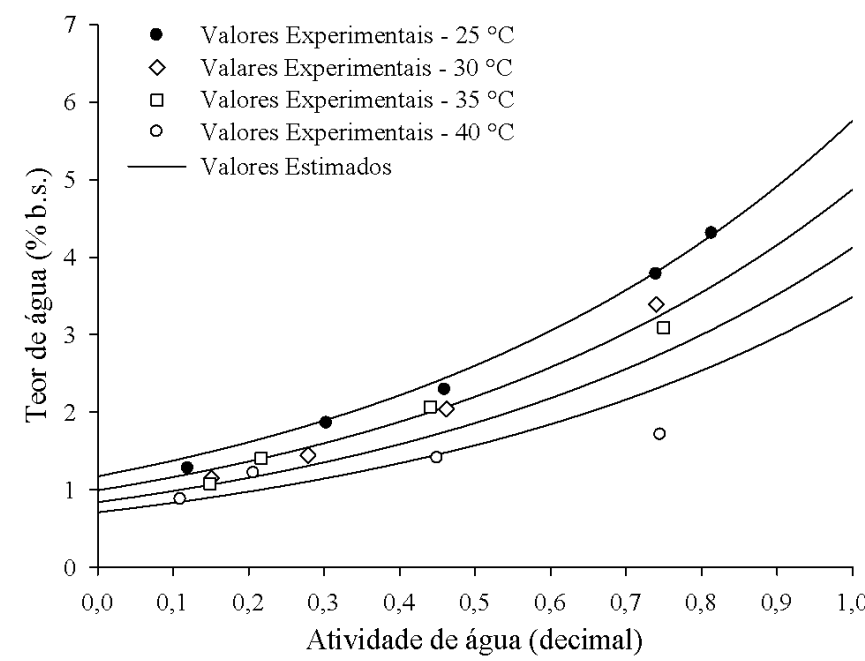

Figura 1. Valores experimentais de teor de água de equilíbrio higroscópico e isotermas de dessorção estimadas pelo modelo de Copace para os frutos de crambe, em diferentes condições de temperatura e atividades de água

As isotermas dos frutos de crambe calculadas pelo modelo de Copace, podem ser classificadas como do tipo III (IUPAC, 1985) conforme verificado para as sementes de nabo forrageiro, por Sousa et al. (2013) que também utilizou o modelo de Copace para a predição do teor de água de equilíbrio higroscópico do produto; apesar disto, para a maioria dos produtos agrícolas as isotermas apresentaram a forma típica sigmoidal (tipo II) como, por exemplo, trigo (Corrêa et al., 2005b), feijão (Phaseolus vulgaris L.) (Resende et al., 2006b), arroz em casca (Iguaz \& Vírseda, 2007) e mamona (Goneli et al., 2008). Segundo IUPAC (1985) as isotermas do tipo III são menos comuns comportamento que indica que os principais constituintes do produto (solutos) apresentam pouca afinidade pelas moléculas de água, como é o caso dos frutos de crambe, que possuem um elevado teor de óleo na sua constituição.

Segundo Henao et al. (2009) as isotermas de sorção são usadas para vários propósitos, como determinação do tempo de secagem ou, ainda, predições para misturar e embalar o produto prevendo as mudanças de umidade que ocorrem durante a armazenagem e, consequentemente, sua estabilidade; de modo geral, a utilização das isotermas de sorção gera informações referentes ao armazenamento adequado e à tomada de decisão sobre medidas preventivas para a manutenção da qualidade do produto.

Na Figura 2 são apresentados os valores do calor isostérico integral de dessorção em função do teor de água de equilíbrio (\% b.s.) estimados de acordo com a Equação.

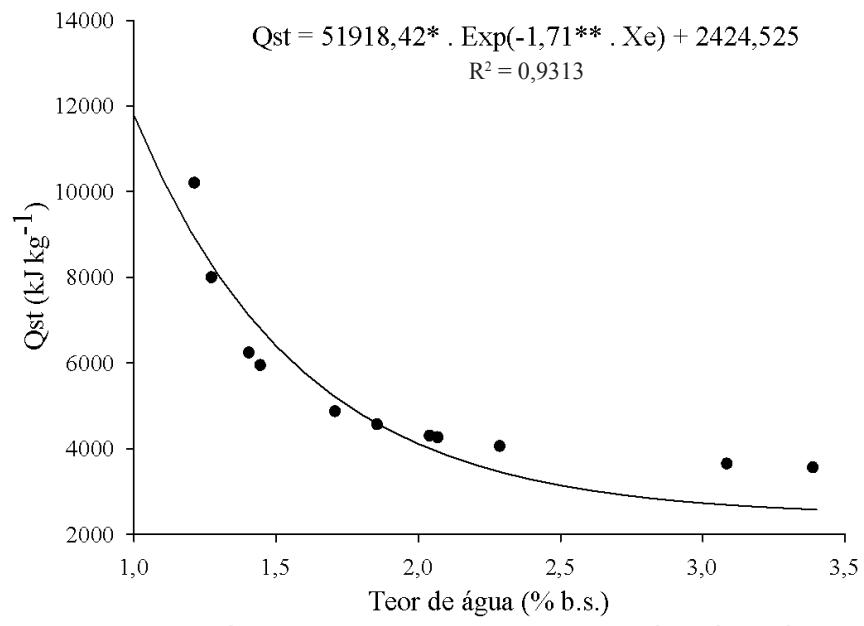

Figura 2. Valores experimentais e estimados do calor isostérico integral de dessorção $\left(\mathrm{Q}_{\mathrm{st}}\right)$ estimados em função do teor de água de equilíbrio dos frutos de crambe

Observa-se que, com a redução do teor de água, ocorre aumento da energia necessária para a remoção de água do produto representada pelos valores do calor isostérico integral de dessorção, conforme observado para diversos produtos agrícolas, como feijão (Resende et al., 2006a), arroz em casca (Resende et al., 2006b), trigo (Corrêa et al., 2005b), noz pistache (Hayoglu \& Gamli, 2007), pimenta malagueta (Silva \& Rodovalho, 2012) e outros. Os valores de calor isostérico integral de dessorção para os frutos de crambe na faixa do teor de água de equilíbrio 1,22 a 3,39 (\% b.s.) variaram de 10.183,33 a $3.538,74 \mathrm{~kJ} \mathrm{~kg}^{-1}$. Ayranci \& Duman (2005) obtiveram, trabalhando com feijão caupi na faixa de teor de água de 4 a 16 (\% b.s) valores de calor isostérico integral de dessorção variando de 3361 a $2472 \mathrm{Kj} \mathrm{kg}^{-1}$, aproximadamente.

Segundo Aviara \& Ajibola (2002) esses resultados confirmam o fato de que nos produtos com maior teor de água a força de ligação entre as moléculas de água e a matéria seca diminui sensivelmente. Tagawa et al. (1993) afirmam que para retirar água de sementes com baixo teor de água é necessário uma quantidade de energia, em média, superior aquela necessária para produtos com elevado teor de água.

\section{ConclusõEs}

1. O teor de água de equilíbrio higroscópico dos frutos de crambe é diretamente proporcional à atividade de água e decresce com o aumento de temperatura, para um mesmo valor de umidade relativa de equilíbrio.

2. Baseando-se em parâmetros estatísticos, os modelos de Copace e Oswin Modificado são os que melhor representam a higroscopicidade dos frutos de crambe quando comparados com os demais modelos testados. 
3. O calor isostérico aumenta com a diminuição do teor de água de equilíbrio sendo necessária uma quantidade maior de energia para retirar a água dos frutos de crambe.

\section{AgRAdECIMENTOS}

Ao Conselho Nacional de Desenvolvimento Científico e Tecnológico $(\mathrm{CNPq})$ e à Coordenação de Aperfeiçoamento de Pessoal de Nível superior (CAPES) pelo apoio financeiro indispensável à execução do presente trabalho.

\section{Literatura Citada}

Araújo, L. F.; Corrêa, P. C.; Silva, R. F. Comparação de modelos matemáticos para descrição das curvas de dessorção de sementes de milho-doce. Pesquisa Agropecuária Brasileira, v.36, p.991-995, 2001.

Aviara, N. A.; Ajibola, O. O. Thermodynamics of moisture sorption in melon seed and cassava. Journal of Food Engineering, v.55, p.107-113. 2002.

Ayranci, E.; Duman, O. Moisture sorption isotherms of cowpea (Vigna unguiculata L. Walp) and its protein isotate at 10, 20 and $30^{\circ} \mathrm{C}$. Journal of Food Engineering, v.70, p.83-91, 2005.

Bastos, S. M. C. Propriedades termodinâmicas de adsorção de água de dois genótipos de arroz vermelho. 2009. 72p. Anápolis: UEG. Dissertação Mestrado

Borges, S.; Borges, E. E. L.; Correa, P. C.; Brune, A. Equilíbrio higroscópico e viabilidade de sementes de angico-vermelho (Anadenanthera peregrina (L.) Speng) em diferentes condições ambientais de armazenamento. Scientia Forestalis, v.37, p.475-481, 2009.

Brasil, Ministério da Agricultura e Reforma Agrária. Secretaria Nacional de defesa Agropecuária. Regras para análise de sementes. Brasília: Mapa/ACS, 2009. 399p.

Brooker, D. B.; Bakker-Arkema, F. W.; Hall, C. W. Drying and storage of grains and oilseeds. Westport: The AVI Publishing Company, 1992. 450p.

Corrêa, P. C.; Goneli, A. L. D.; Resende, O.; Martinazo, A. P.; Botelho, F. M. Comparação entre os métodos estático e dinâmico na determinação do equilíbrio higroscópico das espigas de milho. Revista Brasileira de Produtos Agroindustriais, v.7, p.153-161, 2005a.

Corrêa, P. C.; Goneli, A. L. D.; Resende, O.; Ribeiro, D. M. Obtenção e modelagem das isotermas de dessorção e do calor esotérico de dessorção para grãos de trigo. Revista Brasileira de Produtos Agroindustriais, v.7, p.39-48, $2005 \mathrm{~b}$.

Corrêa, P. C.; Resende, O.; Ribeiro, D. M. Isotermas de sorção das espigas de milho: obtenção e Modelagem. Revista Brasileira de Milho e Sorgo, v.4, p.126-134, 2005c.

Dhingra, O. D.; Sinclair, J. B. Basic plant pathology methods. 2.ed. Boca Raton: CRC Press, 1995. 434p.

Espíndola, L. F. Determinação do teor de água ideal para o armazenamento de sementes de quatro espécies arbóreas ameaçadas da flora brasileira. 61p. Rio de Janeiro: ENBT, 2007. Dissertação Mestrado

Francisco, F. G.; Usberti, R.; Toneli, J. T. C. L. Ajuste de isotermas de sorção de sementes de cultivares de feijoeiro. Revista Brasileira de Sementes, v.29, p.35-39, 2007.
Goneli, A. L. D. ; Corrêa, P. C.; Botelho, F. M.; Oliveira, G. H. H. ; Santos, E. S. Propriedades físicas dos frutos de mamona durante a secagem. Revista Brasileira de Armazenamento, v.33, p. 148-155, 2008.

Goneli, A. L. D. ; Corrêa, P. C. ; Oliveira, G. H. H. ; Botelho, F. M.. Water desorption and thermodynamic properties of okra seeds. Transactions of the ASAE, v.53, p.191-197, 2010.

Guirra, F. Crambe: Uma fonte promissora. Revista Biodieselbr, v.2, p.40-47, 2009.

Hayoglu, I.; GamlI, O. F. Water sorption isotherms of pistachio nut paste. International Journal of Food Science and Technology, v.42, p.224-227. 2007.

Henao, J. D.; Queiroz, M. R.; Haj-Isa, N. M. A. Umidade de equilíbrio de café cereja descascado baseada em métodos estático e dinâmico. Revista Brasileira de Engenharia Agrícola e Ambiental, v.13, p.470-476, 2009.

Iglesias, H.; Chirife, J. Prediction of the effect of temperature on water sorption isotherms of food material. Journal of Food Technology, v.11, p.109-116, 1976.

Iguaz, A.; Vírseda, P. Moisture desorption isotherms of roung rice at high temperatures. Journal of Food Engineering, v.79, p.794-802, 2007.

IUPAC - International Union of Pure and Applied Chemistry. Reporting physisorption data for gas/solid systems with special reference to the determination of surface area and porosity (Recommendations 1984), Pure \& Applied Chemistry, v.57, p.603-619, 1985.

Lima, E. E. de; Silva, A. S. A.; Figueirêdo, R. M. F. de; Queiroz, A. J. de M. Estudo das isotermas e calor isostérico de adsorção da farinha da coroa de frade. Revista Brasileira de Produtos Agroindustriais, v.10, p.163-170, 2008.

Madamba, P. S.; Driscoll, R. H.; Buckle, K. A. Thin-layer drying characteristics of garlic slices. Journal of Food Engineering, v.29, p.75-97, 1996.

Mohapatra, D.; Rao, P. S. A thin layer drying model of parboiled wheat. Journal of Food Engineering, v.66, p.513-18, 2005.

Mortola, V. B.; Meili, L.; Pinto, L. A. A. Determinação das isotermas de equilíbrio para cebola, gelatina e farinha de pescado: Análise dos modelos de GAB, BET e Henderson. Revista Vetor, v.13, p.79-91, 2003.

Neves, M. B.; Trzeciak, M. B.; Vinholes, P. S.; Tillman, A. C.; Villela, F. A. Qualidade fisiológica de sementes de crambe produzidos em Mato Grosso do Sul. In: Simpósio Estadual de Agroenergia, 2007, Pelotas, RS. Anais...Pelotas: EMBRAPA, 2007. p.97-98.

Ordoñez, J. A. Tecnologia de alimentos. Porto Alegre: Artmed, p.25-31, 201-203. 2005.

Resende, O.; Corrêa, P. C.; Goneli, A. L. D.; Martinazzo, A. P; Ribeiro, R. M. Isotermas e calor isostérico de dessorção do arroz em casca. Revista Brasileira de Armazenamento, v.31, p.91-99, 2006a.

Resende, O.; Corrêa, P. C.; Goneli, A. L. D.; Ribeiro D. M. Isotermas e calor isostérico de sorção do feijão. Ciência e Tecnologia dos Alimentos, v.26, p.626-631, 2006b.

Silva, H. W.; Rodovalho, R. S. Isotermas de dessorção das sementes de pimenta malagueta. Global Science and Technology, v.5, p.32-39, 2012. 
Sousa, K. A.; Resende, O.; Costa, L. M. Isotermas de dessorção das sementes de nabo forrageiro obtidas pelos métodos dinâmico e estático. Revista Brasileira de Engenharia Agrícola e Ambiental, v.17, p.216-222, 2013.

Souza, A. D. V.; Vianna, A. C. A.; Soares, C. M.; Ida, E. I. ; Oliveira, L. C. S.; Favaro, S. P. Caracterização química de sementes e tortas de pinhão-manso, nabo-forrageiro e crambe. Pesquisa Agropecuária Brasileira, v.44, p.1328-1335, 2009.
Tagawa, A.; Murata, S.; Hayashi, H. Latent heat of vaporization in buckwheat using the data of equilibrium moisture content. Transactions of the ASAE, v.36, p.113118. 1993.

Wang, N.; Brennan, J. G. Moisture sorption isotherm characteristics of potato at four temperatures. Journal of Food Engineering, v.14, p.269-287, 1991. 